\title{
Chalk stick break
}

\author{
Singh $\mathrm{S}^{1^{*}}$, Bhatt $\mathrm{B}^{2}$, Peredo-Wende $\mathrm{R}^{\mathbf{1}}$ and Kaushik $\mathrm{P}^{\mathbf{1}}$ \\ ${ }^{1}$ Department of Internal Medicine, Albany Medical College, NY, USA \\ ${ }^{2}$ Department of Rheumatology, Crouse Health Hospital, USA
}

\section{Clinical Image}

49-year-old male with diabetes, hypertension, and hyperlipidaemia presented with increasing neck pain, hand paraesthesia, dysesthesia, and gait difficulty with an associated fall from a ladder at work, 2 weeks prior. Prior to his fall, he had been complaining of back pain and limited mobility for many years but had never been seen by a Rheumatologist. Imaging of the cervical spine revealed an extension distraction injury resulting in a "chalk stick" fracture of C6-C7 vertebral bodies (Figure 1). Lateral radiographic view of lumbar spine showing flowing mantles of ossification in the anterior longitudinal ligament from first to the third vertebrae consistent with DISH (Figure 2). Also, there was significant anterior ankylosis of the sacroiliac joints bilaterally, with preservation of the joint space posteriorly (Figure 3 ). The patient lacked any evidence of reactive arthritis or psoriatic arthritis.

Due to cervical canal stenosis and cord signal abnormality, patient required open reduction of C6-C7 dislocation, C6-C7 anterior cervical discectomy and interbody fusion.

Chalk Stick Fractures are fractures of the fused spine, classically seen in Ankylosing Spondylitis. They usually occur through the discovertebral junction in the lower cervical or upper thoracic spine. They may also be seen in diffuse idiopathic skeletal hyperostosis (DISH) or surgical spine fusion. Ankylosing spondylitis or AS can be challengingly difficult to distinguish from diffuse idiopathic skeletal hyperostosis

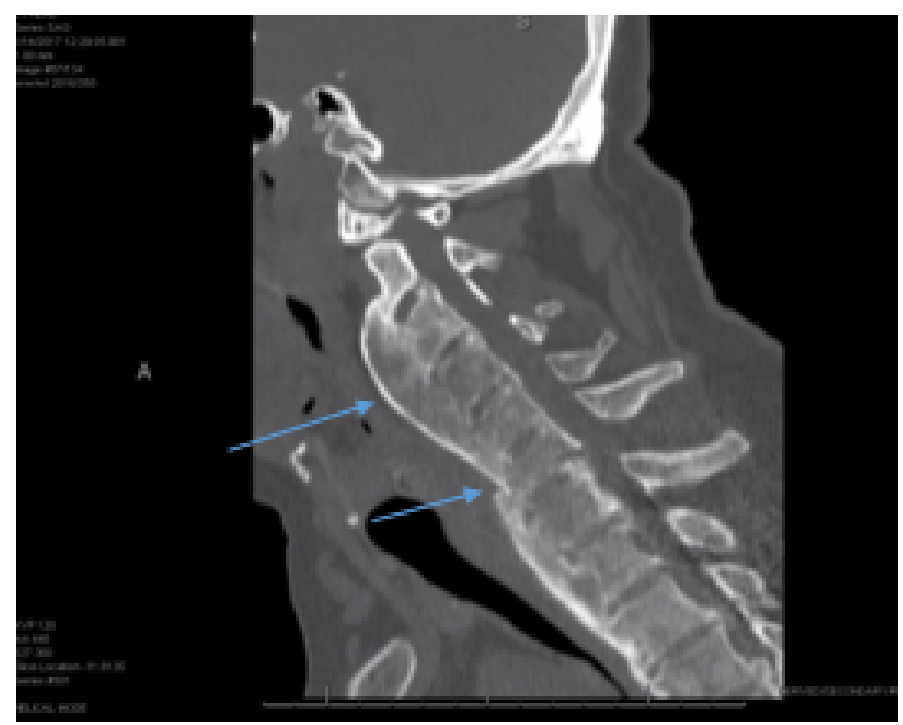

Figure 1. Short Arrow: "chalk stick" fracture at C6-7, Long Arrow: massive flowing ossification

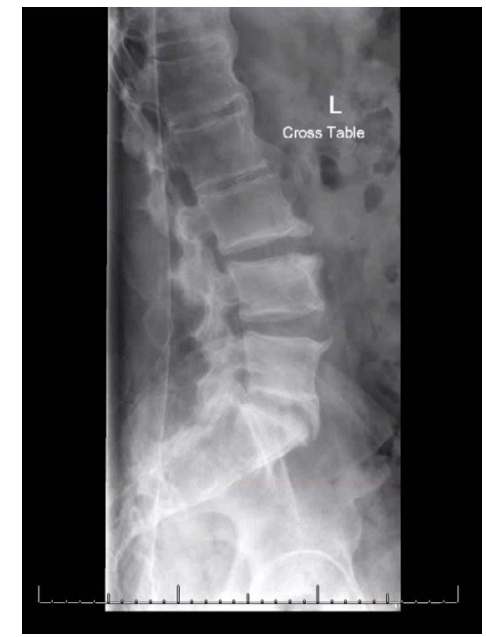

Figure 2. Lateral radiographic view of lumbar spine showing flowing mantles of ossification in the anterior longitudinal ligament from first to the third vertebrae consistent with DISH

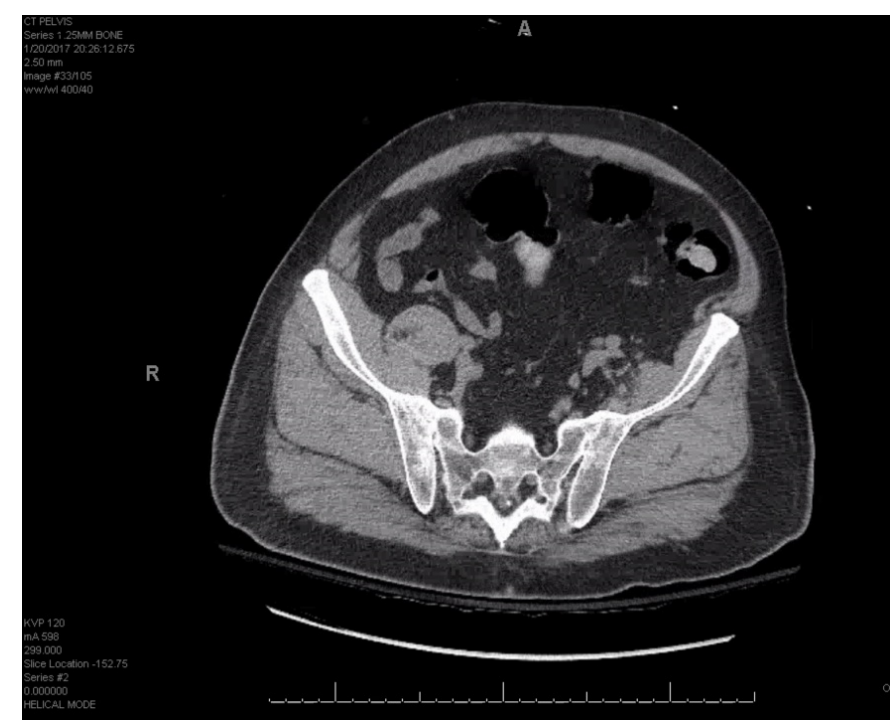

Figure 3. CT scan of the pelvis showing significant anterior ankylosis of the sacroiliac joints bilaterally, with preservation of the joint space posteriorly. No significant joint erosions are noted

*Correspondence to: Kaushik P, Section Chief, Rheumatology service, Department of Internal Medicine, Albany Medical College, USA, Tel: 5186266551; Fax: 5186266606; E-mail: Prashant.kaushik@va.gov

Received: September 15, 2018; Accepted: September 25, 2018; Published: September 27, 2018 
or DISH especially in older individuals [1] with a background of metabolic syndrome. In this case, there was evidence sacroiliitis and HLA B27 testing was positive favouring a diagnosis of AS that had not been picked up for years, along with DISH.

The proposed criteria for DISH should act as guidelines for study purposes and not as rigid requirement for the diagnosis [2]. DISH with SI joint involvement can be distinguished from AS by the absence of sacroiliac joint erosions, the lack of inferior sacroiliac joint fusion and other radiographic and clinical features [3]. Further studies are required to review and modify the criteria to establish the diagnosis of DISH. We further investigated the English literature confirming evidence of DISH and AS coexisting in multiple patients [3-5].

The neo-osteogenesis in AS is leads to excessive new bone formation. However, this bone is abnormal and vulnerable to fractures like in this case where it broke like a 'chalk stick'.

\section{Author Disclosure and Affiliations}

We declare no conflicts of interest.

\section{References}

1. Forestier J, Rotes-Querol J (1950) Senile ankylosing hyperostosis of the spine. Ann Rheum Dis 9: 321-330.

2. Olivieri I, D'Angelo S, Palazzi C, Padula A, Mader R, et al. (2009) Diffuse idiopathic skeletal hyperostosis: differentiation from ankylosing spondylitis. Curr Rheumatol Rep 11: 321-328.

3. Moreno A, Gonzalez M, Duffin M, Lopez-Longo F, Carreno L, et al. (1996) Simultaneous occurrence of diffuse idiopathic skeletal hyperostosis and ankylosing spondylitis. Rev Rheum 63: 292-295.

4. Rillo OL, Scheines EJ, Moreno C, Barreira JC, Porrini JA, et al. (1989) Coexistence of diffuse idiopathic skeletal hyperostosis and ankylosing spondylitis. Clinical Rheumatology 8: 499-503.

5. Durback MA, Edelstein G, Schumacher HR Jr. (1980) Abnormalities of the sacroiliac joints in diffuse idiopathic skeletal hyperostosis: demonstration by computed tomography. J Rheumatology 15: 1506-1511.

Copyright: (C2018 Singh S. This is an open-access article distributed under the terms of the Creative Commons Attribution License, which permits unrestricted use, distribution, and reproduction in any medium, provided the original author and source are credited. 